\title{
Performance Evaluation of Flows with Diverse Traffic and Transmission Rates in IEEE 802.11 WLAN
}

\author{
Yiru Wu, Yinghong Ma, Hongyan Li, Jiandong Li \\ State Key Laboratory of Integrated Service Networks, Xidian University, Xi’an, China \\ Email: yrwu@stu.xidian.edu.cn,yinghongma@xidian.edu.cn, hyli@xidian.edu.cn, jdli@mail.xidian.edu.cn
}

Received July 2013

\begin{abstract}
IEEE 802.11 WLAN cannot guarantee the QoS of applications, thus admission control has been proposed as an essential solution to enhance the QoS. Packet delay and throughput are commonly employed as assessment criterions to determine whether a new connection can be admitted into the WLAN. Considering the real network condition, the analytical model is presented in this paper, which is aimed to evaluate the packet delay and throughput performance of IEEE 802.11 WLAN in nonsaturated conditions, taking into account diverse transmission rates and diverse traffic flows (i.e. flows with different packet sizes and arrival rates) simultaneously. This model is based on Markov chain and the theoretical predictions are verified by simulation in OPNET 14.5. We also analyze the influences of transmission rate diversity and traffic flow diversity on throughput performance. It is observed that, the presence of even one station with lower transmission rate can cause a considerable degradation in throughput performance of all the stations when they have the same packet size and arrival rate. Higher system throughput can be achieved if lower transmission rate stations transmit packets with smaller size or arrival rate.
\end{abstract}

Keywords: IEEE 802.11 DCF; Transmission Rate Diversity; Traffic Flow Diversity; Packet Delay; Throughput Performance

\section{Introduction}

In recent years, the IEEE 802.11 based wireless LANs (WLANs) have gained great popularity due to high bandwidth, low cost and simple deployment. The fundamental mechanism to access the medium in 802.11 is called distributed coordination function (DCF), based on the carrier sense multiple access with collision avoidance (CSMA/ CA) protocol. The contention-based random access nature of the CSMA/CA protocol leads to the result that achieving a satisfactory Quality of Service (QoS) in WLANs is challenging. To enhance QoS support in WLANs, the IEEE 802.11e standard is proposed which introduces prioritization to the legacy DCF by allowing different traffic classes [1]. Nevertheless, 802.11e cannot guarantee the QoS all the time for the existence of contentions among flows of the same priority, especially under heavy load conditions [2].

Admitting a new connection can cause severe degradation in the QoS of the legacy connections in a WLAN, which can be seen from the simulation results by OPNET 14.5. In the simulation, multiple wireless stations were associated with the same $802.11 \mathrm{~b}$ AP, which is connected to a $100 \mathrm{Mbps}$ Ethernet. The setup was used to make full-duplex VoIP calls between a wireless station and a wired station using IP phones. For each call, we used the ITU G711 a-Law codec where frames are sent out every 10 milliseconds. We tested the number of VoIP connection with acceptable voice quality by successively establishing new calls in addition to the ongoing calls. As shown in Figures 1 and 2, as soon as the seventh call was placed, the average packet delay of every VoIP connection became too high and there was a sharp decrease in total throughput.

Admission control has been proposed as an essential

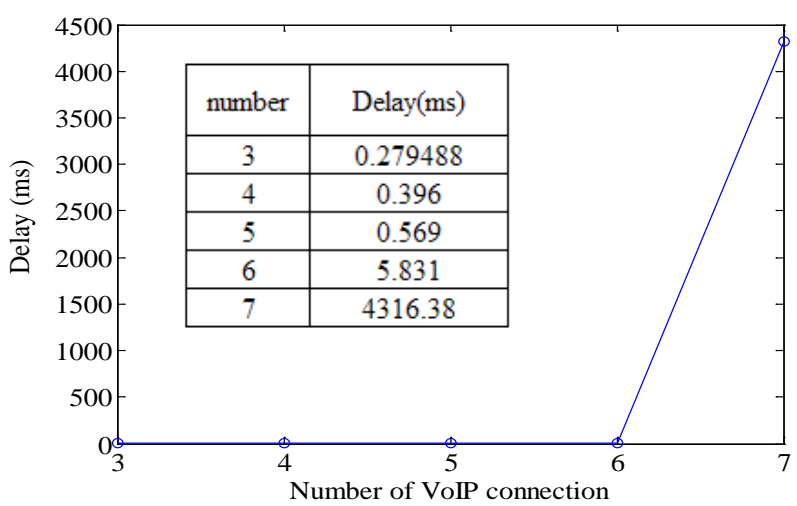

Figure 1. Average packet delay of VoIP connection. 


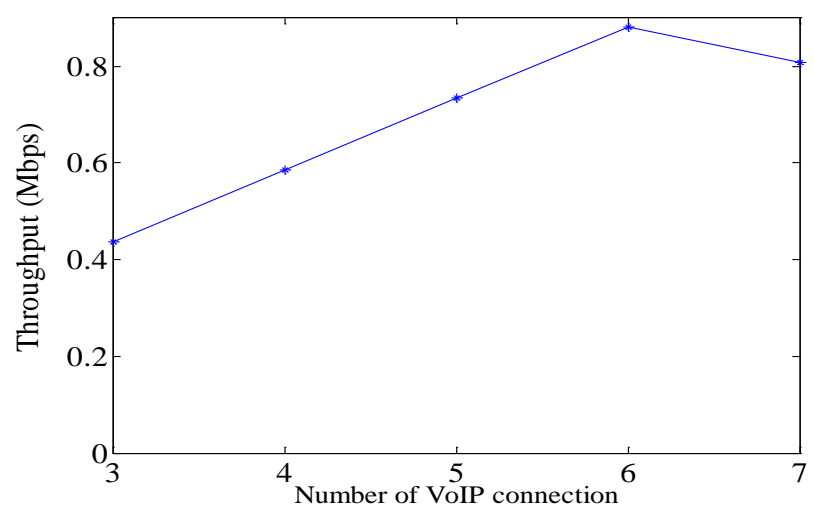

Figure 2. Total throughput.

solution to provide QoS guarantees for applications over WLANs. Packet delay and throughput are commonly employed as assessment criterions to determine whether a new connection can be admitted into the WLAN [3-5, 7]. A loose estimation of delay or throughput is harmful for admission control, because once traffic load exceeds the network capacity, the quality of all ongoing connections will be jeopardized as shown in Figures $\mathbf{1}$ and $\mathbf{2}$. Consequently, the accuracy of predicting delay or throughput is of great significance.

Numerous efforts have been made to model the behavior of the DCF of IEEE 802.11 and analyze the delay and throughput performance of WLAN. Bianchi [6] firstly develops a bidimensional discrete-time Markov chain model to calculate the system throughput in saturated conditions, meaning that the stations always have packets to transmit. The nonsaturated condition is considered in [7-10]. In [7], a modification of [6] is put forward where a probability is introduced that represents a station having no packet ready for transmission. The model is not predictive as this probability is not known as a function of traffic load and must be estimated from simulation. In [8], idle states are added after a successful packet transmission where a station waits for the following packet from upper layers. The delay in the idle states is distributed geometrically with a parameter $\lambda$; nevertheless, no relationship is given between $\lambda$ and the traffic load on the system. In $[9,10]$, the probability of a station having at least one packet ready for transmission is expressed as a function of traffic load, which takes into account the packet arrival rate diversity.

In the previous work, different stations are assumed to have the same transmission rate or packet size for simplification. In order to accurately predicting delay or throughput, we consider the traffic flow diversity (i.e. packet size diversity and arrival rate diversity) and the transmission rate diversity simultaneously, and derive the formulas for delay and throughput in non-saturated cases. Specifically, our contributions presented in this paper are the following,
- We present a discrete Markov chain model to calculate the probability $\tau$ of a station transmitting in an arbitrary time slot in nonsaturated conditions. The Markov chain model utilized considers suspension of the backoff time counter when the channel is sensed busy.

- To determine $\tau$, we express the probability $q$ that represents a station having at least one packet ready for transmission in a randomly chosen time slot. $q$ is related to the length of the time slot which may be occupied by a successful transmission, a collision, or being idle. To precisely calculate the length of the time slot occupied by a collision, we take into account both the packet size diversity and the transmission rate diversity.

- The packet delay is defined as the delay from the time instant when a packet is at the head of the queue to the time instant when the packet is successfully transmitted, which is a stochastic variable. We assume the distribution of packet delay to be a geometric distribution and derive the average packet delay of heterogeneous flows.

- We formulate the individual throughput and system throughput respectively. By simulation in OPNET 14.5, it is verified that these formulas can closely approximate the throughput performance in WLAN.

- We also analyze the influences of transmission rate diversity and traffic flow diversity on throughput performance and draw the conclusion that, the system throughput can be increased if lower transmission rate stations transmit packets with smaller size or arrival rate.

\section{Analytical Model}

In this paper, we consider only the basic access mechanism and assume that: 1) the network consists of $N$ contending stations, labeled $i=1,2, \ldots, N ; 2$ ) packets arrive at the MAC layer of station $i$ based on Poisson process with arrival rate $\lambda_{i}$, where $\left.i \in[1, N] ; 3\right)$ packets of station $i$ have constant size $L_{i}$ and transmission rate $R_{i}$, where $i \in[1, N]$.

Let $T_{S_{i}}$ be the time the channel is sensed busy because of a successful transmission from station $i$; and we have $T_{S_{i}}=L_{i} / R_{i}, i \in[1, N]$. Suppose that there are $M$ different values of $T_{S_{i}}$ (due to the diversity of packet size and transmission rate) labeled $T_{S}^{1}, T_{S}^{2}, \ldots, T_{S}{ }^{M}$ with the relation $T_{S}^{1}>T_{S}^{2}>\cdots>T_{S}^{M}$ holding and that $n^{l}$ stations have the same value of $T_{S_{i}}$ that equals to $T_{S}^{l} \quad(l \in[1, M])$, thus having $\sum_{l=1}^{M} n^{l}=N$.

\subsection{Per-Station Markov Model}

The Markov chain model presented in this paper is depicted in Figure 3. In the model, each station is modeled 


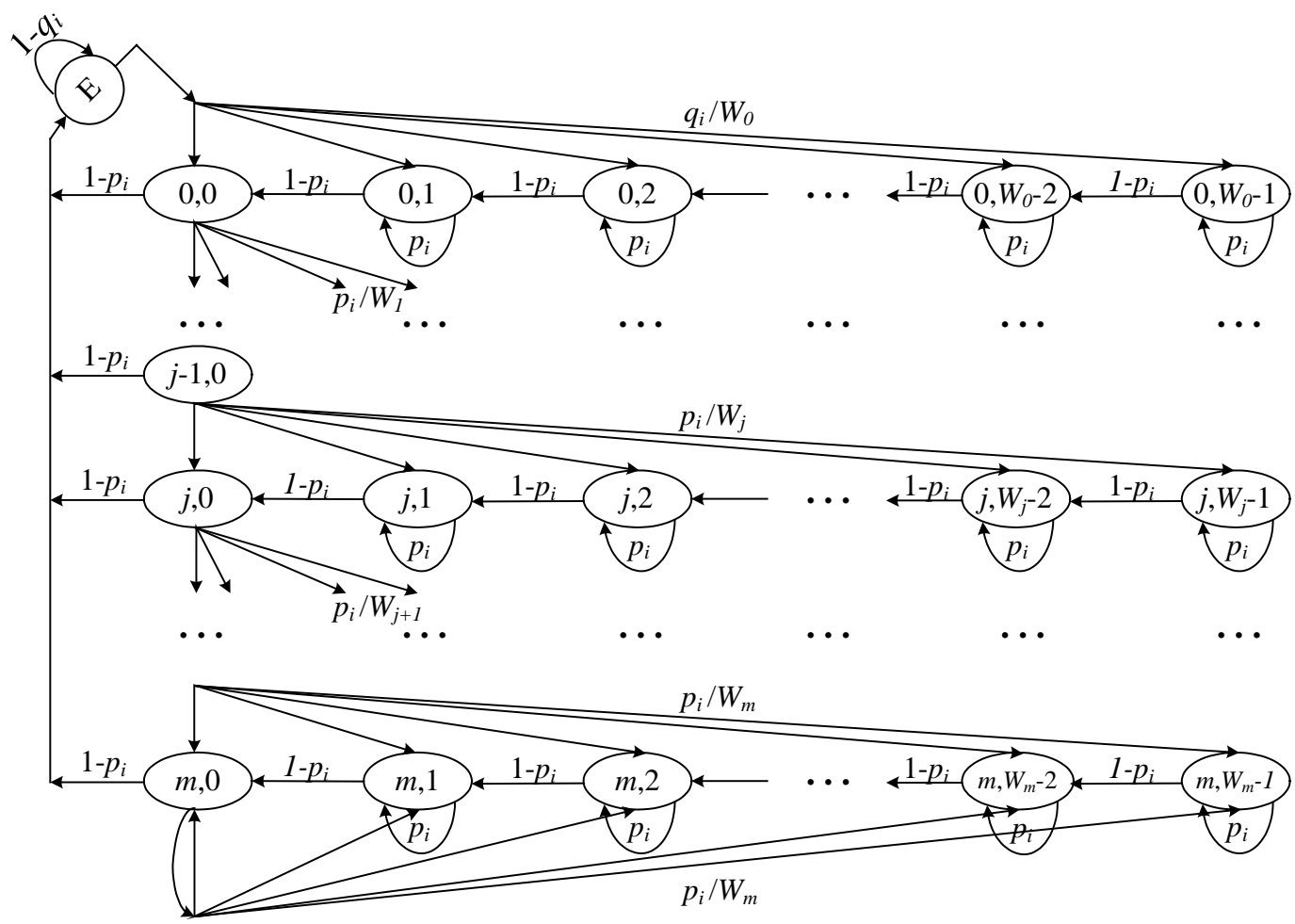

Figure 3. Non-saturated Markov chain model for IEEE 802.11.

by pair of stochastic processes $b(t)$ and $s(t)$, representing the backoff time counter and the backoff stage respectively. For convenience, the simplified notations $(j, k)$ are used instead of $(s(t), b(t))$ to represent each state in this model.

The backoff stage $j$ starts at 0 at the first attempt to transmit a packet and is increased by 1 every time a transmission attempt results in a collision, up to a maximum value $m$. Initially, the backoff time counter $k$ is chosen uniformly between $\left[0, W_{j}-1\right]$, where typically $W_{j}=2^{j} W_{0}$ is the range of the counter and $W_{0}$ is the 802.11 parameter CWmin. At the beginning of each slot, the counter is decremented if the channel is sensed idle and frozen if a transmission is detected on the channel. When the counter reaches zero, the station attempts to transmit.

A new state $E$ is introduced for a station to check whether there is at least one packet to transmit or not after a successful transmission. If there is none packet awaiting transmission, the station remains in this state; otherwise, the 802.11 MAC begins another stage-0 backoff.

We represent by $p_{i}$ the conditional collision probability of a packet transmitted by station $i$, where $i \in[1, N]$. The probability $p_{i}$ is assumed to be constant and independent, regardless of the number of retransmissions already suffered. Under the assumption, we have for $0 \leq k \leq W_{j}-1$

$$
\begin{array}{rlrl}
P[(\mathrm{j}+1, \mathrm{k}) \mid(\mathrm{j}, 0)] & =\frac{p_{i}}{W_{j+1}} & & 0 \leq j<m \\
P[(\mathrm{j}, \mathrm{k}) \mid(\mathrm{j}, 0)] & =\frac{p_{i}}{W_{j}} & j=m \\
P[\mathrm{E} \mid(\mathrm{j}, 0)] & =1-p_{i} & 0 \leq j \leq m
\end{array}
$$

Furthermore, $p_{i}$ also stands for the probability of detecting the channel busy. For $0 \leq j \leq m$ and $0<k \leq W_{j}-1$, we have

$$
\begin{gathered}
P[(\mathrm{j}, \mathrm{k}-1) \mid(\mathrm{j}, \mathrm{k})]=1-p_{i} \\
P[(\mathrm{j}, \mathrm{k}) \mid(\mathrm{j}, \mathrm{k})]=p_{i}
\end{gathered}
$$

$q_{i}$ represents the probability of a station having at least one packet ready for transmission. We have for $0 \leq k \leq W_{0}-1$

$$
\begin{array}{r}
P[\mathrm{E} \mid \mathrm{E}]=1-q_{i} \\
P[(0, \mathrm{k}) \mid \mathrm{E}]=\frac{q_{i}}{W_{0}}
\end{array}
$$

Let $b_{i, j, k}$ be the stationary distribution of the Markov chain for station $i$. We can obtain a closed-form solution for this chain. First, note that

$$
\begin{aligned}
& b_{i, j-1,0} \cdot p_{i}=b_{i, j, 0} \rightarrow b_{i, \mathrm{j}, 0}=p_{i}^{j} b_{i, 0,0} \quad 0<\mathrm{j}<m \\
& b_{i, \mathrm{~m}-1,0} \cdot p_{i}=\left(1-p_{i}\right) b_{i, \mathrm{~m}, 0} \rightarrow b_{i, \mathrm{~m}, 0}=\frac{p_{i}^{m}}{1-p_{i}} b_{i, 0,0} \\
& \sum_{\mathrm{s}=0}^{m} b_{i, \mathrm{~s}, 0} \cdot\left(1-p_{i}\right)=b_{E} \cdot q_{i} \rightarrow b_{E}=\frac{b_{i, 0,0}}{q_{i}}
\end{aligned}
$$


Owing to the chain regularities, the following relations hold, for $0<k \leq W_{j}-1$

$$
b_{i, j, k}=\frac{W_{j}-k}{W_{j}} \cdot \frac{1}{1-p_{i}} \cdot \begin{cases}\left(1-p_{i}\right) \sum_{\mathrm{s}=0}^{m} b_{i, \mathrm{~s}, 0} & j=0 \\ p_{i} \cdot b_{i, j-1,0} & 0<j<m \\ p_{i} \cdot\left(b_{i, \mathrm{~m}-1,0}+b_{i, m, 0}\right) & j=m\end{cases}
$$

$$
\begin{gathered}
{ }_{m}^{m} \text { From relations } \quad \begin{array}{c}
\text { (4), we can obtain } \\
\sum_{\mathrm{j}=0} b_{i, j, 0}=
\end{array} b_{i, 0,0} /\left(1-p_{i}\right) \text {. Then rewrite (5) as } \\
b_{i, j, k}=\frac{W_{j}-k}{W_{j}} \cdot \frac{1}{1-p_{i}} \cdot b_{i, j, 0} \quad 0 \leq j \leq m, \quad 0<k \leq W_{j}-1
\end{gathered}
$$

Thus, all the stationary probabilities $b_{i, j, k}$ can be expressed in terms of $b_{i, 0,0}$, and $b_{i, 0,0}$ is finally determined by imposing the normalization condition

$$
\sum_{j=0}^{m} \sum_{k=0}^{W_{j}-1} b_{i, j, k}+b_{E}=1
$$

from which

$$
\begin{aligned}
& b_{i, 0,0} \\
& =\frac{2 q_{i}\left(1-2 p_{i}\right)\left(1-p_{i}\right)^{2}}{q_{i}\left(1-2 p_{i}\right)\left(W_{0}+1\right)+q_{i} p_{i} W_{0}\left(1-\left(2 p_{i}\right)^{m}\right)+2\left(1-2 p_{i}\right)\left(1-p_{i}\right)^{2}}
\end{aligned}
$$

As any transmission attempt occurs when $k=0$, irrespective of the backoff stage, we can now express the probability $\tau_{i}$ that station $i$ transmits in a randomly chosen slot time, that is

$$
\begin{aligned}
\tau_{i} & =\sum_{\mathrm{j}=0}^{m} b_{i, j, 0}=\frac{b_{i, 0,0}}{1-p_{i}} \quad 2 q_{i}\left(1-2 p_{i}\right)\left(1-p_{i}\right) \\
& =\frac{q_{i}\left(1-2 p_{i}\right)\left(W_{0}+1\right)+q_{i} p_{i} W_{0}\left(1-\left(2 p_{i}\right)^{m}\right)+2\left(1-2 p_{i}\right)\left(1-p_{i}\right)^{2}}{}
\end{aligned}
$$

A collision occurs if more than one station is transmitting in the same time slot. As station $i$ transmits with probability $\tau_{i}$, the conditional collision probability can be expressed as

$$
p_{i}=1-\prod_{\substack{u=1 \\ u \neq i}}^{N}\left(1-\tau_{u}\right)
$$

With $\mathrm{m}$ and $W_{0}$ given in 802.11 standard, in order to determine $\tau_{i}$ and $p_{i}$, we must determine $q_{i}$ first.

\subsection{Probability $q$ with Traffic Flow Diversity and Transmission Rate Diversity}

Under our assumption of Poisson process for packet arrival, the probability $q_{i}$ can be expressed as

$$
q_{i}=1-e^{-\lambda_{i} E_{S}}
$$

where $E_{S}$ is the average length of a time slot. The length of the virtual time slot is not a fixed value, and each time slot may be occupied by a successful transmission, a collision, or the medium being idle, which gives

$$
E_{S}=P_{i d l e} \cdot \sigma+\sum_{i=1}^{N} P_{S_{i}} T_{S_{i}}+\sum_{l=1}^{M} P_{c}^{l} T_{c}^{l}
$$

where, $\sigma$ is the duration of an empty slot time; $P_{\text {idle }}$ is the probability the channel is sensed idle (i.e., none station transmitting);

$$
P_{i d l e}=\prod_{u=1}^{N}\left(1-\tau_{u}\right)
$$

$T_{S_{i}}$ is the time the channel is sensed busy because of a successful transmission from station $i ; P_{S_{i}}$ is the probability station $i$ successfully transmits (i.e., only station $i$ transmitting);

$$
P_{S_{i}}=\tau_{i} \prod_{\substack{u=1 \\ u \neq i}}^{N}\left(1-\tau_{u}\right)
$$

$T_{c}^{l}$ is the time the channel is sensed busy during a collision, i.e., the longest transmission time of stations involved in a collision; then $T_{c}^{l}$ has $\mathrm{M}$ possible values that are

$$
T_{c}^{l}=T_{S}^{l} \quad l \in[1, M]
$$

$P_{c}{ }^{l}$ is the probability of $T_{c}{ }^{l}$ being equal to $T_{S}{ }^{l}$, for $l \in[1, M]$

$$
P_{c}^{l}=\sum_{j=1}^{n^{l}} \prod_{i=1}^{N_{0}+j-1}\left(1-\tau_{i}\right) \cdot \tau_{N_{0}+j} \cdot\left[1-\prod_{i=N_{0}+j+1}^{N}\left(1-\tau_{i}\right)\right]
$$

where $N_{0}=\sum_{k=1}^{l-1} n^{k}$.

The set of Equations (9)-(11) $(i=1,2, \ldots, N)$ represent a nonlinear system with $3 N$ unknowns $\tau_{i}, p_{i}$ and $q_{i}$, which can be solved by numerical techniques.

\subsection{Average Packet Delay}

We approximate the random length of the virtual time slot by its average value $E_{S}$ given by (12). Suppose $X_{i}$ is a random variable that represents the number of time slots that station $i$ needs for a successful transmission, then the packet delay of station $i$ is also a random variable given by $T_{i}=E_{S} \cdot X_{i}$. Assume that the distribution of $X_{i}$ is a geometric distribution, and that the probability of station $i$ successfully transmitting in a random slot is $P_{S_{i}}$ given by (14). Thus, the distribution of packet delay is a geometric distribution given as follows,

$$
P\left\{T_{i}=E_{s} \cdot x_{i}\right\}=\left(1-P_{S_{i}}\right)^{x_{i}-1} \cdot P_{S_{i}}
$$

from which the average packet delay of station $i, E\left[T_{i}\right]$, 
derives,

$$
E\left[T_{i}\right]=E_{S} \cdot E\left[X_{i}\right]=E_{S} \cdot \frac{1}{P_{S_{i}}}
$$

\subsection{Throughput Formulation}

We are now able to express the throughput of station $i$ as the ratio of the time that the medium is occupied by station $i$ for successful transmission to the average packet delay of station $i$.

$$
S_{i}=\frac{T_{S_{i}}}{E\left[T_{i}\right]}=\frac{P_{S_{i}} T_{S_{i}}}{E_{S}}
$$

As a result, the system throughput is

$$
S=\sum_{i=1}^{N} S_{i}
$$

\section{Model Verification}

Suppose there are 6 stations and two possible transmission rates of $1 \mathrm{Mbps}$ and $11 \mathrm{Mbps}$ in the WLAN. The other parameters are summarized in Table 1.

We first evaluate the throughput when different stations have different transmission rates but the same packet size $L_{i}=L=1024$ bytes (including MAC, IP, UTP and RTP headers) and the same arrival rate $\lambda_{i}=\lambda=100$ packets/sec. Starting with all stations at 1 Mbps, we increase the transmission rate of one of them to $11 \mathrm{Mbps}$ in each step. Eventually all six stations have the rate of $11 \mathrm{Mbps}$. We simulate this setup in OPNET 14.5. Figure 4 shows that the analytical formulas (19) and (20) can closely approximate the individual throughput and the system throughput respectively. It is obvious from the figure that even one lower transmission rate can cause a considerable degradation in throughput performance of all the stations. This degradation is a consequence of the DCF mode which guarantees equal packet transmission probability to all stations. As a result, lower data rate stations receive more time to transmit and unfairly bring down the throughput of the higher data rate stations. This degradation was previously observed in [11] under saturated conditions. Then we exploit different packet sizes and arrival rates in the analysis.

Table 1. System parameters.

\begin{tabular}{cc}
\hline Slot Time & $20 \mu \mathrm{s}$ \\
\hline PHY header & $192 \mu \mathrm{s}$ \\
Propagation Delay & $1 \mu \mathrm{s}$ \\
DIFS & $50 \mu \mathrm{s}$ \\
SIFS & $10 \mu \mathrm{s}$ \\
ACK & 112 bit + PHY header \\
CWmin & 32 \\
$\mathrm{~m}$ & 5 \\
\hline
\end{tabular}

On the one hand, we reduce the packet size of stations with rate $1 \mathrm{Mbps}$ to $L^{\prime}=102$ bytes, but the packet arrival rate remains to be 100 packets/sec. As seen from Figure 5, the system throughput and throughput of 11 Mbps stations are improved, whereas the 1 Mbps stations are worse off. In this case, lower data rate stations and higher data rate stations receive the same amount of time to transmit.

On the other hand, we reduce the packet arrival rate of stations with rate $1 \mathrm{Mbps}$ to $\lambda^{\prime}=15$ packets/sec, but the packet size remains to be 1024 bytes. The throughput performance plotted in Figure 6 resembles that in Figure $\mathbf{5}$, which shows that the throughput performance of system and high data rate stations can be enhanced by sacrificing that of low data rate stations. By reducing the packet arrival rate of lower data rate stations, the packet transmission probability of lower rate stations is reduced.

Figure 7 shows the throughput performance when 1

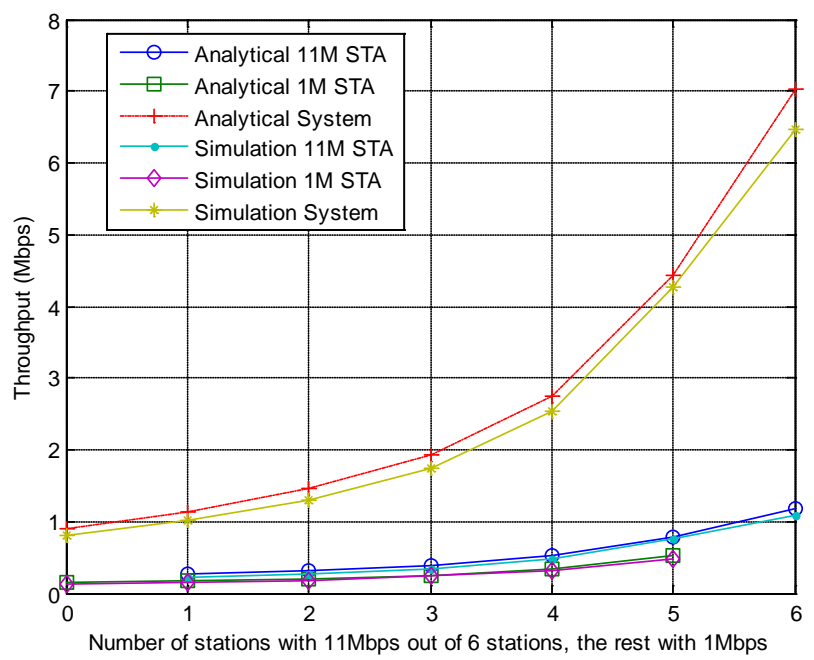

Figure 4. Throughput with transmission rate diversity $(L=$ 1024 bytes, $\lambda=100$ packets/sec).

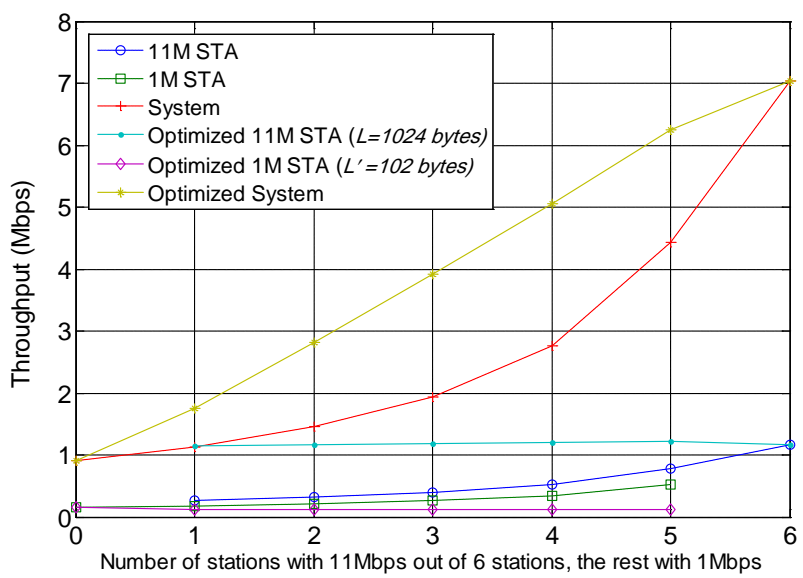

Figure 5. Throughput with diversity of transmission rates and packet sizes $(\lambda=100$ packets/sec $)$. 


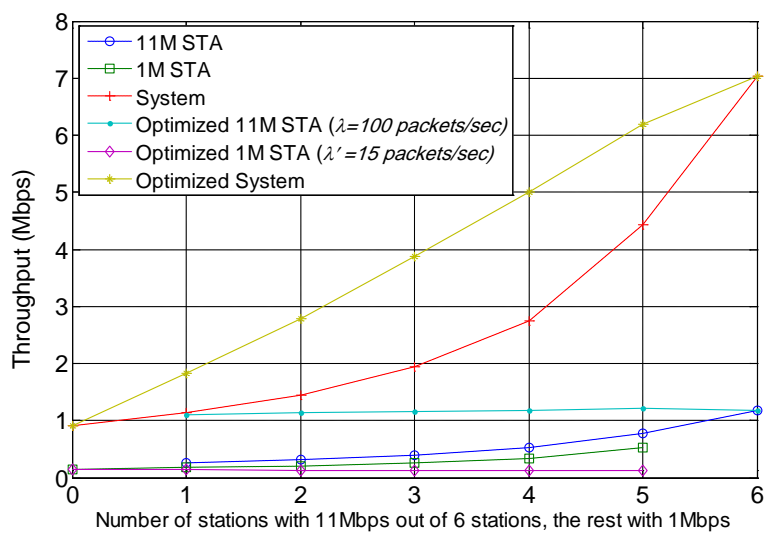

Figure 6. Throughput with diversity of transmission rates and packet arrival rates $(L=1024$ bytes).

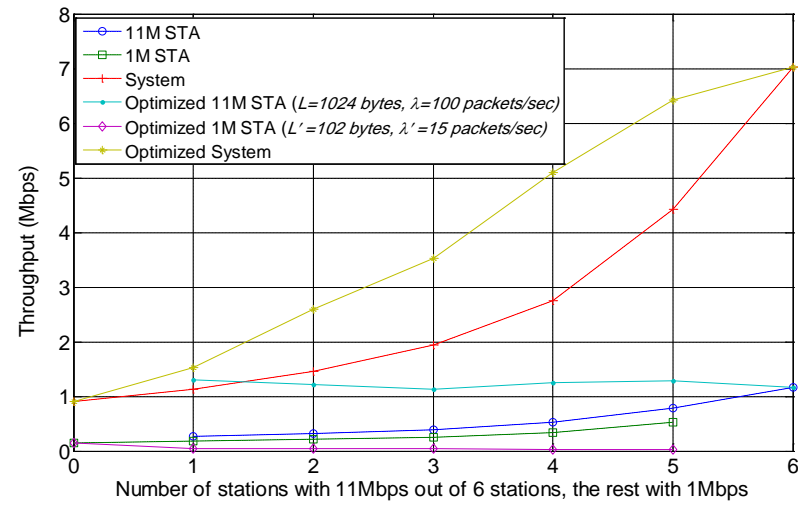

Figure 7. Throughput with diversity of transmission rates and traffic flows.

Mbps stations have the packet size 102 bytes and the arrival rate 15 packets/sec. There is no striking enhancement in throughput performance, compared to that in Figures 5 and $\mathbf{6}$.

\section{Conclusion}

In this paper, we have presented an analytical model to evaluate the packet delay and throughput performance of IEEE 802.11 WLAN in nonsaturated conditions. Simulation and analysis results show that our analytical formulas for throughput can closely approximate the performance for different transmission rates and traffic flows. The analysis results also show that higher system throughput can be achieved if lower data rate stations transmit packets with smaller size or arrival rate. Furthermore, this evaluation method can easily be utilized with sufficient accuracy in admission control in the real network environment.

\section{Acknowledgments}

This work is supported by the National Science Foundation (60972047, 61231008), National S\&T Major Project
(2011ZX03005-004, 2011ZX03004-003, 2011ZX03005003-03, 2013ZX03004007-003), Shannxi 13115 Project (2010ZDKG-26), National Basic Research Program of China(2009CB320404), Program for Changjiang Scholars and Innovative Research Team in University (IRT0852), the 111 Project (B08038) and State Key Laboratory Foundation (ISN 1002005, ISN090305).

\section{REFERENCES}

[1] IEEE 802.11e WG, "Wireless LAN Medium Access Control (MAC) and Physical Layer (PHY) Specifications: Medium Access Control (MAC) Enhancements for Quality of Service (QoS),” IEEE std 802.11e-draft ed, 2005.

[2] J. Villalón, P. Cuenca and L. Orozco-Barbosa, “On the Capabilities of IEEE 802.11e for Multimedia Communications over Heterogeneous 802.11/802.11e WLANs," Springer Science, Vol. 36, 2007, pp. 27-38.

[3] L. Lin, H. Fu and W. Jia, "An Efficient Admission Control for IEEE 802.11 Networks Based on throughput Analyses of (Un) Saturated Channel," IEEE GLOBECOM, 2005, pp. 3017-3021.

[4] A. Bazzi, M. Diolaiti and G. Pasolini, "Measurement Based Call Admission Control Strategies in Infrastructured IEEE 802.11 WLANs,” IEEE International Symposium on Personal, Indoor and Mobile Radio Communications, Vol. 3, 2005, pp. 2093-2098.

[5] A. Abdrabou and W. Zhuang, "Stochastic Delay Guarantees and Statistical Call Admission Control for IEEE 802.11 Single-Hop Ad Hoc Networks," IEEE Transactions on Wireless Communications, Vol. 7, No. 10, 2008, pp. 3972-3981.

http://dx.doi.org/10.1109/T-WC.2008.070564

[6] G. Bianchi, "Performance Analysis of the IEEE 802.11 Distributed Coordination Function," IEEE Journal on Selected Areas in Communications, Vol. 18, No. 3, 2000, pp. 535-547. http://dx.doi.org/10.1109/49.840210

[7] M. Ergen and P. Varaiya, "Throughput Analysis and Admission Control for IEEE 802.11a,” Mobile Networks and Applications, Vol. 10, No. 5, 2005, pp. 705-716. http://dx.doi.org/10.1007/s11036-005-3364-9

[8] G.-S. Ahn, A. T. Campbell, A. Veres and L.-H. Sun, "Supporting Service Differentiation for Real-Time and Best-Effort Traffic in Stateless Wireless Ad Hoc Networks (SWAN)," IEEE Transaction on Mobile Computing, Vol. 1, No. 3, 2002, pp. 192-207.

http://dx.doi.org/10.1109/TMC.2002.1081755

[9] D. Malone, K. Duffy and D. Leith, "Modeling the 802.11 Distributed Coordination Function in Nonsaturated Heterogeneous Conditions," IEEE/ACM Transactions on Networking, Vol. 15, No. 1, 2007, pp. 159-172. http://dx.doi.org/10.1109/TNET.2006.890136

[10] Y. Xu and C. Xu, "On Traffic Flow Diversity over IEEE 802.11 Ad Hoc Networks,” IEEE International Conference on Wireless Communications, Networking and Mobile Computing, 2011, pp. 1-4.

[11] M. Ergen and P. Varaiya, "Formulation of Distributed Coordination Function of IEEE 802.11 for Asynchronous 
Networks Mixed Data Rate and Packet Size," IEEE

Transaction on Vehicular Technology, Vol. 57, 2008, pp. 